\title{
Supporting Productive Integration of Web 2.0-Mediated Collaboration
}

\author{
Mun Fie Tsoi \\ National Institute of Education Singapore, Nanyang Technological University, \\ 1 Nanyang Walk, Singapore 637616 \\ raymond.tsoi@nie.edu.sg
}

\begin{abstract}
This paper describes a research evidence-based practice model, TSOI Hybrid Learning Model as a viable alternative to support productive integration of Web 2.0-mediated collaboration for learning. The model is advanced from the Science learning cycle and the Kolb's experiential learning cycle. An authentic example on understanding multimedia learning pedagogy for pre-service teachers in chemistry education is illustrated. The model guides the learning design involving Web 2.0-mediated collaborative activities. Outcomes in terms of richness of collaborative learning and reflections have been positive. Implications will be discussed in the context of blended learning in science education.
\end{abstract}

Keywords: Hybrid learning model, Web 2.0, collaborative learning.

\section{Introduction}

This paper provides an insight into applying a research evidence-based practice model to support productive integration of Web 2.0-mediated collaboration for learning. To address the issues on the use of social software tools in education for example, quality learning, course design and delivery cited by Franklin \& van Harmelen [1], Mason \& Rennie [2], Minocha [3], an alternative pedagogical framework to integrate Web 2.0 such as Blogs and Wikis productively for learning is described. As such, having this in mind, the TSOI Hybrid Learning Model $^{\mathrm{TM}}$ \& $\odot 2005$ All rights reserved. (TSOI HLM) can serve as a useful support to integrate productively Web 2.0-mediated collaborative activities.

It is envisaged that as in previous studies on its functions and applications conducted by Tsoi [4], Tsoi [5], the learning model will contribute as an innovative approach for a productive integration of Web 2.0-mediated collaboration for learning.

\section{Conceptual Framework of TSOI HLM}

The TSOI HLM is advanced from the Science learning cycle model and the Kolb's experiential learning cycle. The term hybrid will mean the mixing of two different things to give a better product which in this case is a learning model that is pedagogically more innovative and comprehensive than each of the original model namely, the 
Science learning cycle model and the Kolb's experiential learning cycle model. It is inclusive since it also encompasses the characteristics of each original model. The Science learning cycle model being inquiry-based represents an inductive application of information processing models of teaching and learning as reported by Karplus [6], Lawson [7], Renner \& Marek [8]. It has three phases in a cycle: exploration, concept invention, and concept application as shown in Figure 1. The exploration phase focuses on "What did you do?" while the concept invention phase places emphasis on "What did you find out?". The concept application phase entails the application of the concept.

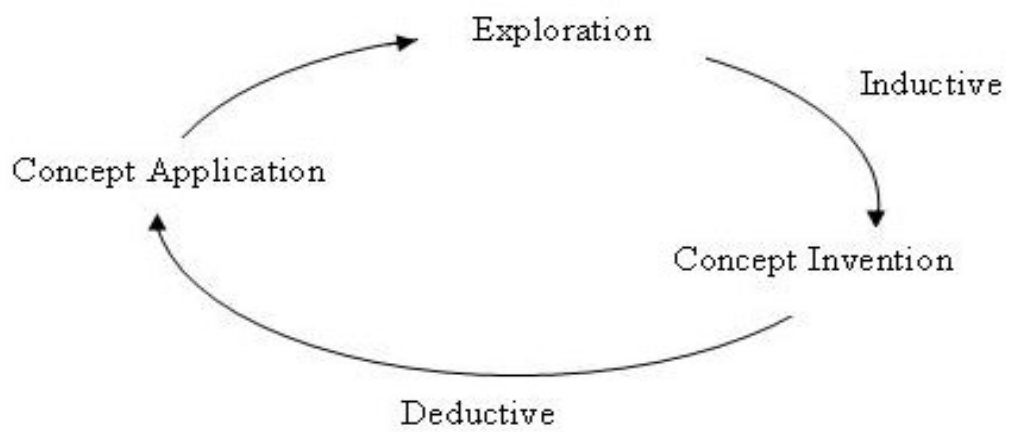

Fig. 1. The Science learning cycle model

The exploration phase (gathering of data) is often accomplished during a science activity or an experiment that is usually performed in the science laboratory. During this exploration phase, learners learn through their own actions and reactions in a new situation and have the opportunity to explore new learning materials and new ideas with minimal guidance from the teacher.

The concept invention phase gives the opportunity to the student and/ or teacher to derive the concept from the data through classroom discussion. This phase involves the introduction of a new term or terms. Ideally, learners are encouraged to discover as much of a new pattern as possible before the term is revealed to them. The third phase, concept application allows the student to explore the relevance and application. This concept application phase is essential as it allows learners to extend the range of applicability of the new concept.

The Kolb's experiential learning cycle researched by Kolb [9] as shown in Figure 2 represents learning as a process in a cycle of four stages, namely, concrete experience, reflective observation, abstract conceptualization, and active experimentation. The concrete experience stage is about "doing" while the reflective observation stage concerns the "understanding the doing". The abstract conceptualization stage focuses on the "understanding" part and the active experimentation stage is about "doing the understanding". This experiential learning cycle model has also been used by His \& Agogino [10], Tsoi \& Goh [11] as a framework for organizing interactive multimedia learning activities. 
Kolb also created four quadrants in his model of experiential learning. He named each quadrant a learning style as diverger, converger, assimilator or accommodator (see Figure 2).

\section{Concrete Experience}

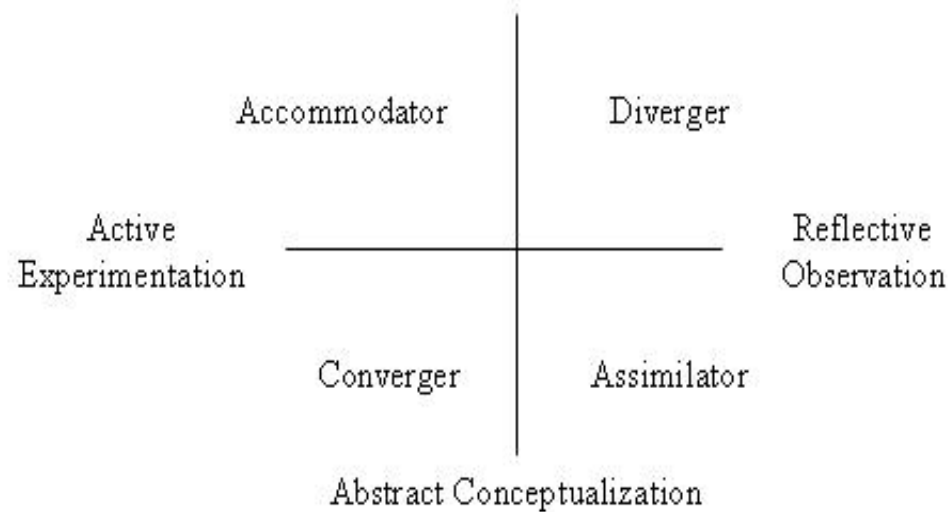

Fig. 2. Kolb's Experiential Learning Model (Smith and Kolb, 1986, p16)

For convergers, experience is grasped through abstract comprehension and transformed through action, which combines abstract conceptualization and active experimentation. For divergers, experience is grasped as opposite of convergers, that is, concretely through feelings and transformed through thought, which combines concrete experience with reflective observation. For assimilators, experience is grasped through abstract comprehension and transformed through thought, which combines abstract conceptualization and reflective observation. For the accommodators, experience is grasped concretely through feelings and transformed by action, combining the features of concrete experience and active experimentation.

The TSOI Hybrid Learning Model $^{\mathrm{TM}} \& \odot^{\circ} 2005$ All rights reserved, conceptualized by Tsoi [12], Tsoi [13] represents learning as a cognitive process in a cycle of four phases: Translating, Sculpting, Operationalizing, and Integrating. One of the key features is to promote active cognitive processing in the learner for meaningful and engaged learning proceeding from inductive to deductive learning. Besides, it is inclined towards constructivism. Figure 3 shows the four phases of this learning model.

The Translating phase is similar to the exploration phase of Science learning cycle model and the concrete experience stage of Kolb's experiential learning cycle model. This is where interactive experiences are translated to beginning ideas or concepts to be further engaged in the Sculpting phase. The Sculpting phase parallels the concept invention phase of Science learning cycle model and predominantly the reflective observation stage of the Kolb's experiential learning cycle including partially the abstract conceptualization stage of the Kolb's experiential learning cycle. This is where the beginning idea or concept still in its raw form is further molded to a concrete form that is meaningful to the learner. 


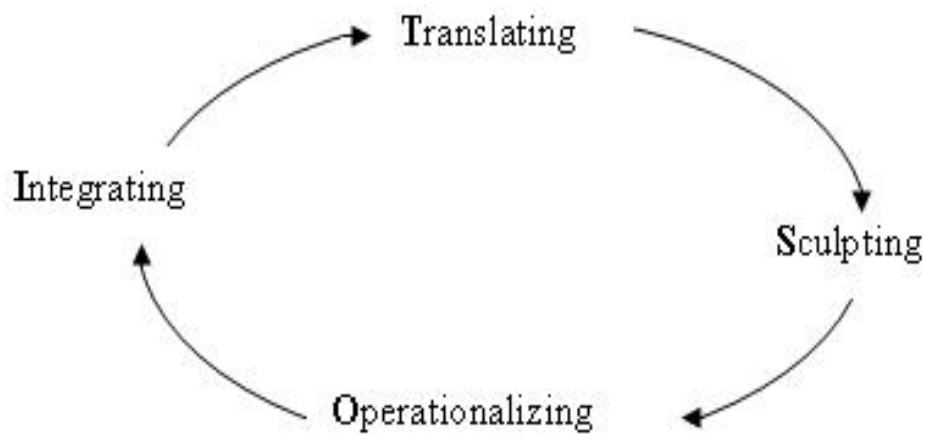

Fig. 3. TSOI Hybrid Learning Model $^{\mathrm{TM} \& @} 2005$ All rights reserved

The Operationalizing phase similar to predominantly the abstract conceptualization stage of the Kolb's experiential learning cycle involves increasing the understandings of the relationship between thinking and concept acquisition. The Integrating phase parallels the concept application of Science learning cycle model as well as the active experimentation stage of Kolb's experiential learning cycle. This is where the concept is applied to new domains in which the transfer of learning is practiced.

\section{Integration Support for Web 2.0-Mediated Collaboration}

The hybrid learning model guides the learning design implemented by the author as the researcher that involves participatory learning as well as integrates Web 2.0 such as blogs and wikis for collaboration. Face to face interactions for a preliminary experience on multimedia learning and its relation to TSOI HLM are realized in the Translating phase while a multimedia learning chemistry module designed based on the TSOI HLM as the pedagogic model is embedded in an e-learning environment for the Sculpting phase. In this phase, group collaboration for the construction of the critical attributes or features of the concept of this hybrid learning model is accomplished in the form of blogs. The Operationalizing phase focuses on interactive group activities on applications of this hybrid learning model to lesson planning of other topics. This is for internalizing purpose. Implications on the use of this hybrid learning model in teaching and learning in science education are completed individually in the form of wikis for the Integrating phase. This learning design will provide the pedagogical support needed for productive integration of Web 2.0-mediated collaborative activities. An example of a curriculum studies course on understanding multimedia learning pedagogy in chemistry education for 162 pre-service teachers of the PGDE (S) course (Postgraduate in Diploma in Education, Secondary) is used.

\subsection{Integration Support: Translating Phase}

During the first phase, the Translating phase, the instructional learning activity though general in nature, is designed to have an initial relationship to the principle underlying 
the concept which to be further engaged in the second phase, the Sculpting phase. The Translating phase emphasizes concept initial exposure for preliminary experience.

In the Translating phase, face to face interactions infused with cooperative learning strategies such as think and pair share, round table and number heads together are carried out in 2 sessions of 2 hours each in the form of group discussions. For example, responses to a question on "what do you understand by the term multimedia learning" are elicited and discussed. The idea is to give an opening experience to what does multimedia learning mean to them. The next main activity focuses on the fundamentals of TSOI HLM as the pedagogic model for multimedia learning design to prepare the trainee teachers for the Sculpting phase. As such, the beginning idea of the TSOI HLM will be further engaged in the Sculpting phase.

In chemistry education, stoichiometry, cited by Tsoi, Goh \& Chia [14] as an abstract and difficult topic is used to illustrate the understanding and applications of the hybrid learning model. One of the subtopics used is solution concentration. This next section, the sculpting phase will provide insights on the application of the TSOI HLM, as an example to demonstrate its use as a pedagogic model for designing this multimedia learning module.

\subsection{Integration Support: Sculpting Phase}

During the second phase, the Sculpting phase, knowledge of the concept is beginning to be constructed based on the learner's facilitated multimedia experiences from the Translating phase as well as the learner's guided multimedia experiences from the Sculpting phase. The concept still in its beginning or raw form as taken from the Translating phase is logically sculpted or shaped to a more concrete form by a series of appropriate and relevant instructional learning activities that are designed meaningfully to assist the learner to identify the critical attributes of the concept. As such, the third phase, the Operationalizing phase is important as it can provide the opportunity for the learner to acquire a complete picture of all the required critical attributes of the concept for conceptual understanding and internalization. The Sculpting phase emphasizes concept construction for its critical attributes.

The development of solution concentration concept consists of four instructional learning episodes in accordance to the four phases of the TSOI HLM. The following subsections describe these four instructional learning episodes which are (a) Which is more concentrated, (b) Physical Meaning and Definition, (c) Investigating Chemical Reactions, and (d) A Simple Equation.

The translating phase is illustrated to show part of the TSOI HLM since the focus of this paper is not on designing a multimedia learning module. The first instructional learning episode," Which is more concentrated" as a Translating phase provides the learner with visual representations in which the relationship between concentration of the solution and the amount of solute particles dissolved in the solution will be formulated cognitively. In the first activity, the learner is asked to compare 2 solutions in terms of concentration which involves the amount of solute particles dissolved in the solution. Observational response is elicited. This is then extended to the second activity where the learner is posed a question as to how to make the concentration of solution A the same as solution B (see Fig 4). These visual representations act as a 
beginning idea or concept of solution concentration which is further molded in the next Sculpting phase of TSOI HLM, Physical Meaning and Definition.

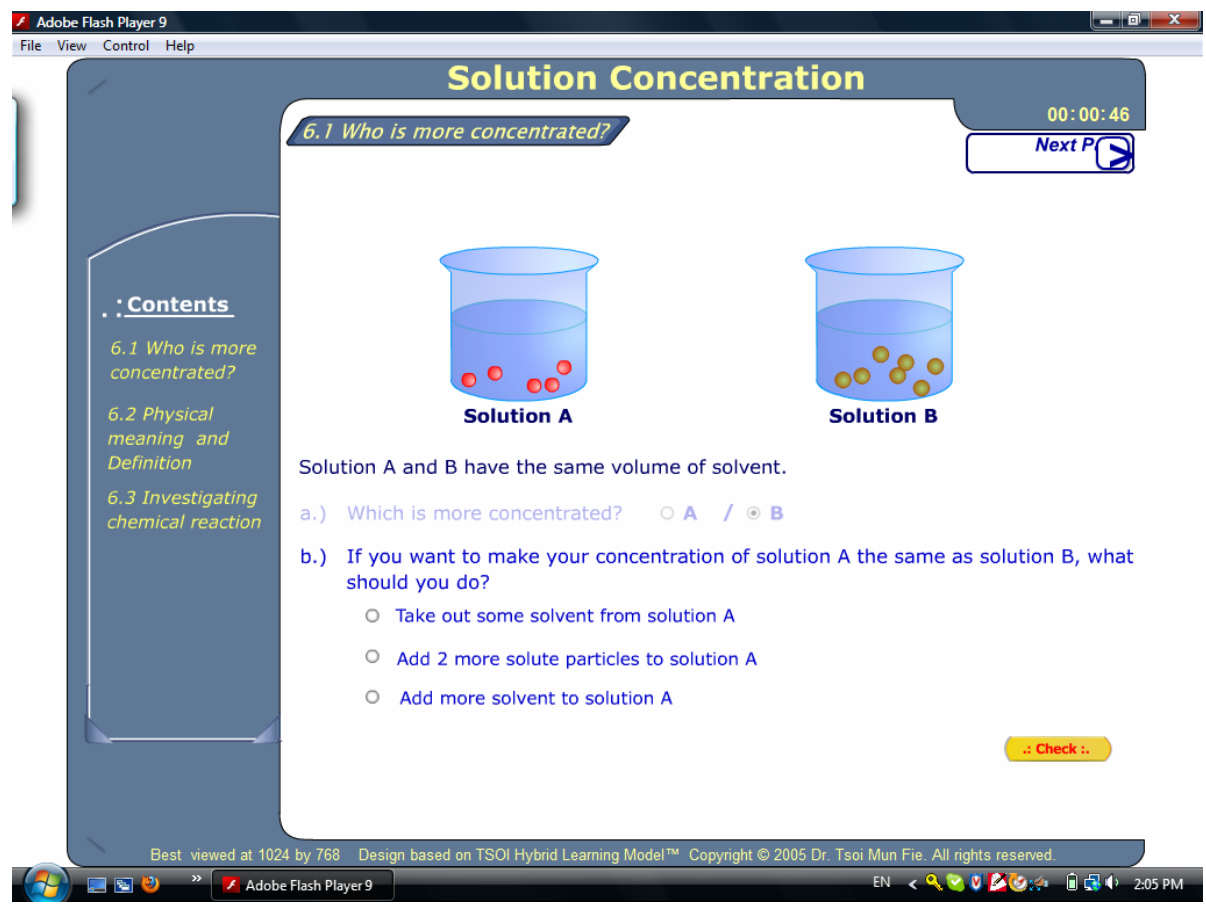

Fig. 4. Solution Concentration Multimedia Learning Module (Translating phase)

The individual learner is given a week to observe on how the multimedia learning module is developed based on the TSOI HLM as its pedagogic model and reflect on it using a journal article as a reference. After which, the group members are given a week to discuss their observations using Web 2.0 technology such as the blog tool and summarize their group discussions. The blog tool is used for its ease in providing comments and also the pre-service teachers prefer to use the blog tool than the discussion forum. Essentially, the understanding of the hybrid learning model in terms of its critical attributes or features is constructed here. A sample of a group blog is shown in Figure 5. A summary of some samples of group blog contents is shown in Figure 6.

\subsection{Integration Support: Operationalizing Phase}

The third phase, the Operationalizing phase is crucial as it serves as the vital bridge connecting the Sculpting phase and the Integrating phase for not only concept formation but also concept internalization in which all the critical attributes of the concept are linked together so as to prepare the learner to be operationally ready for further applications in the Integrating phase. The Operationalizing phase emphasizes concept internalization for its meaningful functionality. 
In the Operationalizing phase, the group members collaborate in a 2 hour session to provide the design of a chemistry lesson for secondary school level based on the hybrid learning model. At the end of the collaborative process, the group members take turns to present their works to the class for comments in a mode of gallery walk. These classroom activities are for the internalization process.

\section{QCY520_Tsoi(Aug09):The Teaching of Chemistry I (permalink)}

\section{Summary TG04 - Group of 4 (names removed) (permalink)}

Created on Thursday, 10/08/2009 3:29 PM by ._name removed From the contributions of our group members, we conclude that the e-learning module on concentration has employed the TSOI $^{\oplus}$ hybrid learning model effectively

Translating Phase

- Engage students' prior knowledge; Exploration of raw forms of concepts

- Engage students in learning, Use of visual, impactful and authentic visual aids

- Issue of assessment of prior knowledge of the students, "Correctness" of students' prior knowledge

Sculpturing Phase

- Logical sequence of content, Meaning and definition of concentration was brought across by first introducing the concept of homogenous solutions

- Developing the method to calculate the number of moles from a given volume of given concentration of solution, Facilitates learner reflection with appropriate pauses

- Students actively involved in thinking process, Crucial in determining the critical attribute of the concept

- Addressing of common misconception: What happens to the concentration of a solution when I draw out $50 \mathrm{~cm}^{3}$ from the $100 \mathrm{~cm}^{3}$ of solution? We feel that it was a good question to test if the students really understood that the concentration is not only dependent on the amount of solute but the volume of solvent as well

Operationalizing Phase

- Partially-worked problems and finally practice problems, Repeated for other problems. Connection between the symbolic representation (equations) and the microscopic level (particles shown) is illustrated.

- Facilitate students to internalize the knowledge as they are shown the links between various concepts, which they will be able use in the next phase.

- The students also start to learn more about problem solving processes. Students are slowly brought through different types of questions based on varying the 4 factors of mole, mass, concentration and volume.

Integrating Phase

- Apply what they learnt from the previous three units to novel situations and problems in this last phase of the $\mathrm{TSOI}^{\odot}$ model

- Integrate what they have learnt so far into different contexts such as purity, titration/volumetric analysis, neutralization and dilution problems, Meaningful learning as students can apply their knowledge constructed thus far to other situations/contexts, Enhances the learning process since the students are able to use the knowledge they have learnt almost immediately

Fig. 5. A sample of a group blog 


\section{Concluding Thoughts:}

In conclusion, our personal opinion is that each step of the TSOI's Learning Model is highly crucial. It is all interconnected and to go to the next stage in the construction of conceptual knowledge, there must be a systematic flow of stages. The interactive program promotes cognitive thinking on the students' part and allows them to actively participate in the activity. Applying the concepts and principles of "concentration" from simpler examples to more difficult ones also helps students to develop their knowledge on the topic in a more structured manner, hence it is easier for students to understand and integrate the knowledge. Overall, the experience has indeed been very fruitful for us.

\section{Summary}

Basically, my group has learnt that the TSOI hybrid learning model consists of 4 stages, with the first stage being one where visualizations are used, that students are able to make sense of the new concept they're being exposed to. Subsequently, the process builds up with worked examples provided at every stage, such that the new concept they are learning becomes more concretized and their knowledge of the topic is further built up. They should finally arrive at the place where they can then apply what they have learnt into situations they have not encountered before with whatever examples they have been exposed to. Incorporating both Piaget's and Kolb's learning models, the TSOI hybrid learning model makes the whole process of acquiring and processing new knowledge more complete \& meaningful.

\section{Summary}

The Tsoi Hybrid Learning model is very effective in getting students to understand difficult concepts by going through the four phases in sequence; Translating, Sculpting, Operationalizing and Integrating. This gradual increase in students' thoughts is very much in line with van Hiele's model since the stages are hierarchical and students cannot skip a stage.

In the first phase Translation, students use visualization and analysis to translate their prior knowledge to what they see.

In the Sculpting Phase, students construct knowledge and when provoked with questions, followed by pauses students are allowed to generate critical ideas. And like van Hiele level 1, the students are beginning to reason inductively.

In the Operationalizing phase, the students begin to understand the relationships between the ideas and concepts generated to form an abstract idea of the processes occurring. This is also possible through problem solving processes.

In the Integrating phase, students form a network of concepts and ideas, summarizing them so as to envision the bigger picture. The learned concepts can therefore now be effectively put to practice in different context.

As such, the TSOI Hybrid Learning Model allows for systematic and logical reasoning of concepts such that learning of these concepts becomes naturally embedded to students' schemata. And in this way, learning is meaningful.

\section{Comments}

The model provides a systematic breakdown of how subjects can taught effectively, highlighting the crucial cognitive processes that take place. It is noted that the model does not only apply to chemistry, but also other subjects as well.

To conclude, the TSOI Hybrid Learning model is a useful framework for teachers to use when planning the instructional process. It is particularly useful in chemistry as many concepts are often abstract and would require a well-structured thought process for learning to be meaningful. Overall, we have learnt (1) how multimedia can be employed to provide interactive and concrete experiences for learners to build their conceptual understanding (2) how the TSOI model is a helpful guideline for teachers to plan their lessons to make learning of abstract concepts more meaningful and effective for our students.

Fig. 6. Some samples of blog contents 


\subsection{Integration Support: Integrating Phase}

During the fourth phase, the Integrating phase emphasizes concept application for meaningful transfer of knowledge. In the Integrating phase, the Wiki tool is used within a week for personal reflections. The individual is asked to reflect and provide up to 6 points its implication on teaching and learning of chemistry as well as think \& describe briefly of how you as a teacher can apply this hybrid learning model as a practice framework to guide you in your teaching of chemistry concepts. A sample of an individual wiki is shown in Figure 7.

\section{QCY520_Tsoi(Aug09):The Teaching of Chemistry I (permalink)}

\section{6 points of implications on teaching and learning of using Tsoi's model (permalink) last} edited by . name removed on Sunday, 10/25/2009 12:50 PM

1) This model allows teachers to be able to teach Chemistry in a systematic way. Chemistry concepts are usually quite abstract and to enhance the learning efficiency of the students, it is important that teachers use the model to guide and reinforce the concept in a step by step manner or students can get quite lost.

2) Many teachers and students are not able to teach or learn the concepts in Chemistry in such a way that they are able to use the concept and apply in other situations. This model took that into consideration and allows teachers or students to be able to teach or learn to apply the concepts to different situations.

3) This model allows students to be active learners in the teaching process as it enables students to be able to explore and form the concepts, form linkages with other concepts and to learn on how to apply them in everyday life.

4) However it is important this process not get too guided. As in the example of the concentration, every single step is guided in the calculation and answers to questions posed can be easily obtained by clicking on the tab. Students are spoon fed instead of becoming active learners in this case.

5) The duration spent and emphasis for each phase should vary according to the ability of the students. For example, longer time and more emphasis might be place in the translating and sculpting phase for students in normal academic and technical as they are usually weaker in their concepts. However for the higher ability students, more emphasis should be on the operationalizing and internalizing phase because they are able to catch on the concepts faster and too much time spent in the beginning stages will bore them.

6) Although this is a good basic framework for teachers to build their lessons upon, it is important in real life application of this model that teachers do not become too religious with it. Lessons are dynamic and smooth flowing if teachers are too insistent on following every stage religiously, the efficiency of learning will be lost.

How can I use this in the classroom?

To me personally, I really enjoy using this framework to guide my lesson planning. It is easy to use and enables us to teach in a systematic manner. Many a times, teachers are lost in how to introduce a concept that is difficult or abstract and this framework helps them to be able to break it down different steps that they can present easily and students will be able to learn efficiently as well. However it is important that I learn to be flexible with this model. Although the model was introduced in a stage by stage manner, it need not be taught rigidly in this way. I have to vary the time and effort needed on each stage based on the ability of the students as well.

Fig. 7. A sample of an individual wiki entry 


\section{Discussions}

The Translating phase of the TSOI HLM is a significant phase as it presents the learner an initial preliminary awareness of the concept which in this case is about a pedagogical model for teaching and learning to be learned. In other words, the experiences be it in form of an e-learning module or face-to-face mode facilitated are translated to a beginning concept by the learner. As such, it is essential to first identify the critical attributes of the concept to be learnt so that varied activities can be designed to assist the learner to identify these critical attributes and eventually leading to acquisition of concept mastery. The instructional activities experienced by the learner in the Translating phase should be familiar to the learner so that one can make connections to one's existing knowledge structures. Following the realization of the Translating phase, the learner's preliminary experience is then given more meaning in the Sculpting phase.

During the second phase, the Sculpting phase of the TSOI HLM, the beginning concept experienced still in its raw form is logically shaped to a more concrete form by a series of appropriate and relevant instructional learning activities that are "crafted" meaningfully to assist the learner to identify the critical attributes of the concept to be learned. These instructional learning activities are also designed to encourage the learner to be actively involved in the appropriate thinking processes, for example, abstracting, and observing, comparing and identifying patterns, predicting that the learner needs to accomplish to determine the critical attributes of the concept. As such, it is appropriate and productive to integrate Web 2.0 such as blogs for collaborative learning.

The Operationalizing phase is the central bridge connecting the Sculpting phase and the Integrating phase particularly in the aspect of concept internalization. There is an important need for the concept that is already constructed to be internalized for meaningful functionality. Besides, an awareness of the problem solving processes is also established. This also applies to group collaboration on the design of lessons to consolidate. During the fourth phase, the Integrating phase, the just learned concept already internalized is then applied to new situations thereby building external connections with other prior concepts as well. The concept is also integrated in different contexts in order for meaningful learning to occur. As the concept learned is a pedagogical model, it is relevant and productive to integrate Wiki, Web 2.0. in the learning process.

The outcomes of the process of integration of Web 2.0-mediated collaborative activities in terms of the richness of the contents of the blogs and wikis have been encouraging and positive to some extent. A sample of how the critical attributes of the hybrid learning model have been constructed using Blogs, Web 2.0 is shown in Figure 5 and 6. This has demonstrated richness of the blog contents in the group's efforts in their discussion and comments. Further to this use of blog tool, the Wiki tool, Web 2.0 for purpose of integrating the concept of the learning model is also well utilized by the pre-service teachers. A sample of the thought process is shown in Figure 7. Indeed, excerpts from the Web 2.0 responses lend support to the potential of the hybrid learning model for productive integration of Web 2.0-mediated collaboration. Besides, studies by Tsoi $[12,15]$ have found a statistically significant difference between pretest and posttest achievement means at the .05 level as they pertain to a learner's level of conceptual understanding of mole concept for each of the four groups using a multimedia learning package for learning of the mole concept, which has as its pedagogic model the TSOI hybrid learning model. It has been likely that the 
four phases of the hybrid learning model together as a whole entity also has a positive overall effect on the conceptual learning of mole concept.

In this preliminary study, the author recognizes limitation that lies partly in the research rigour of the process for example, empirical validation. However, this sets the groundwork for studying this alternative way of approaching the practice of integrating Web 2.0mediated collaboration productively. In essence, the TSOI Hybrid Learning Model has the functional potential capacity to give the educator an alternative practice model for productive integration of Web 2.0-mediated collaboration. The learner will build on the concrete experience, and will learn how to create knowledge and integrate the knowledge with existing ideas and concepts in other context and more importantly, to be an active learner engaged in the various learning processes including collaborative learning.

\section{References}

1. Franklin, T., van Harmelen.: Web 2.0 for learning and teaching in higher education. Report, the Observatory of Borderless Higher Education, London (2007)

2. Mason, R., Rennie, F.: E-learning and Social Networking Handbook. Routledge, New York (2008)

3. Minocha, S.: Role of social software tools in education: a literature review. Education \&Training 51, 353-369 (2009)

4. Tsoi, M.F.: Designing e-learning cognitively: TSOI Hybrid Learning Model. International Journal of Advanced Corporate Learning 1, 48-52 (2008)

5. Tsoi, M.F.: Applying TSOI hybrid learning model to enhance blended learning experience in science education. Interactive Technology and Smart Education 6, 223-233 (2009)

6. Karplus, R.: Teaching and the Development of Reasoning. University of California Press, Berkeley (1977)

7. Lawson, A.E.: Science Teaching and the Development of Thinking. Wadsworth, Belmont (1995)

8. Renner, J.W., Marek, E.A.: An educational theory base for science teaching. Journal of Research in Science Teaching 27, 241-246 (1990)

9. Kolb, D.: Experiential Learning: Experience as the Source of Learning and Development. Prentice Hall, Englewood Cliffs (1984)

10. His, S., Agogino, A.M.: The impact and instructional benefit of using multimedia case studies to teach engineering design. Journal of Educational Multimedia and Hypermedia 3, 351-376 (1994)

11. Tsoi, M.F., Goh, N.K.: Practical multimedia design for chemical education. In: Breaker, J., et al. (Series eds.) \& G. Cumming et al. (Vol eds.) Advanced Research in Computers and Communications in Education: New human abilities for the networked society., vol. 1, pp. 946-949. IOS Press, The Netherlands (1999)

12. Tsoi, M.F.: Designing for Engaged e-learning: TSOI Hybrid Learning Model. The International Journal of Learning 15, 225-232 (2008)

13. Tsoi, M.F.: Designing for Engaging: Hybrid Learning Model. The International Journal of the Computer, the Internet and Management 15, 29.1-29.4 (2007)

14. Tsoi, M.F., Goh, N.K., Chia, L.S.: Some suggestions for the teaching of the Mole Concept. In: Wass, Margit (eds.) Enhancing Learning: Challenge of Integrating Thinking and Information Technology into the Curriculum, vol. 2, pp. 778-785. Educational Research Association, Singapore (1998)

15. Tsoi, M.F.: Development and effects of multimedia design on learning of mole concept. Published doctoral thesis, Nanyang Technological University, Singapore (2007) 